\title{
Kernos
}

Revue internationale et pluridisciplinaire de religion grecque antique

$27 \mid 2014$

Varia

\section{Roberta RIZzo, Culti e miti della Sicilia antica e protocristiana}

\section{Nicola Cucuzza}

\section{(2) OpenEdition \\ Journals}

\section{Edizione digitale}

URL: http://journals.openedition.org/kernos/2252

DOI: $10.4000 /$ kernos. 2252

ISSN: 2034-7871

\section{Editore}

Centre international d'étude de la religion grecque antique

Edizione cartacea

Data di pubblicazione: 1 novembre 2014

Paginazione: 483-484

ISBN: 978-2-87562-055-2

ISSN: 0776-3824

\section{Notizia bibliografica digitale}

Nicola Cucuzza, « Roberta rizzo, Culti e miti della Sicilia antica e protocristiana », Kernos [En ligne], 27 | 2014, mis en ligne le 12 novembre 2014, consulté le 22 septembre 2020. URL : http:// journals.openedition.org/kernos/2252 ; DOI : https://doi.org/10.4000/kernos.2252

Questo documento è stato generato automaticamente il 22 settembre 2020.

Kernos 


\title{
Roberta RIzzo, Culti e miti della Sicilia antica e protocristiana
}

\author{
Nicola Cucuzza
}

\section{NOTIZIA}

Roberta RIZzo, Culti e miti della Sicilia antica e protocristiana, Roma, Salvatore Sciascia Editore, 2012. 1 vol. $16 \times 24$ cm, 400 p. ISBN : 978-88-8241-387-3.

1 Cento anni dopo il volume di E. Ciaceri, Culti e miti nella storia dell'antica Sicilia (Catania, 1911), l'opera di Roberta Rizzo si presenta come un utile strumento di studio per chi voglia conoscere il versante siciliano della mitologia antica. Più di trecento lemmi, disposti in ordine alfabetico e divisi in capitoli per ciascuna lettera dell'alfabeto, trattano di divinità ed eroi che in qualche modo - più o meno direttamente - sono connessi alla mitologia ed ai culti documentati nella grande isola mediterranea (p.13242): oltre alle più generali notizie relative a genealogie e miti, sono infatti ricordati $i$ dati letterari, ma anche epigrafici, numismatici ed archeologici specificamente legati alla Sicilia. Ogni singolo lemma è corredato da note che rimandano alla fonte (antica o moderna) da cui sono desunte le informazioni: queste rinviano alla corposa bibliografia (p. 257-324) ed all'ampio elenco di fonti antiche utilizzate (p.327-348). Chiude il volume una serie di indici: delle voci, dei nomi di personaggi reali e mitici menzionati nelle singole voci, dei luoghi (antichi e moderni) ed infine delle cose notevoli.

2 Come si desume dalle pagine introduttive - che seguono la Prefazione di Antonino Buttitta (p. 5-8) -, l'obiettivo dello studio è quello di fornire "una mappa della cultura religiosa della Sicilia antica fino all'affermarsi del cristianesimo" (p.9). In verità, contrariamente a quanto lascerebbe intendere il titolo del volume, la trattazione riservata al cristianesimo si limita ad osservazioni relative alla continuità di prassi cultuali riscontrabili in alcuni casi fra le religioni pagane e quella cristiana. Inoltre, malgrado l'inserimento in una collana di studi archeologici, il taglio stesso dell'opera e la specifica sensibilità dell'A. portano a privilegiare nettamente l'utilizzo di fonti 
letterarie piuttosto che archeologiche. Il carattere essenzialmente divulgativo dell'opera giustifica qualche semplificazione (come quella del passaggio dalle pratiche cultuali pagane a quelle cristiane), alcune sviste ed omissioni bibliografiche (quasi inevitabili in un lavoro di così ampia portata, condotto da un'unica studiosa). Meno chiaro risulta l'inserimento di alcune annotazioni, come quella, ad esempio, della testa di Gorgone nella moderna bandiera della Regione siciliana (p. 134, 140, 226, 230). Anche se l'uso delle note a piè di pagina piuttosto che alla fine di ogni capitolo sarebbe stata preferibile da parte del lettore, l'articolazione in lemmi in ordine alfabetico facilita la consultazione, mentre l'indice dei nomi permette di rintracciare agevolmente le notizie riguardanti le figure che non sono state oggetto di una specifica trattazione.

Il volume, come detto, costituisce in ogni caso un prezioso punto di riferimento per chi voglia conoscere i miti della Sicilia antica: oltre che per il più vasto pubblico di appassionati, la ricchezza della documentazione analizzata lo renderà senza dubbio un utile strumento di lavoro anche per gli specialisti.

\section{AUTORI}

NICOLA CUCUZZA

Università degli studi di Genova 